Claremont Colleges

Scholarship@ Claremont

All HMC Faculty Publications and Research

HMC Faculty Scholarship

1-1-1989

\title{
Continuum Model of Thin-Film Deposition and Growth
}

Andrew J. Bernoff

Harvey Mudd College

Seth Lichter

University of Arizona

\section{Recommended Citation}

A. J. Bernoff and S. Lichter, A Continuum Model of Thin Film Deposition," Phys. Rev. B 39 (1989) 10560-10569. doi: 10.1103/ PhysRevB.39.10560

This Article is brought to you for free and open access by the HMC Faculty Scholarship at Scholarship @ Claremont. It has been accepted for inclusion in All HMC Faculty Publications and Research by an authorized administrator of Scholarship @ Claremont. For more information, please contact scholarship@cuc.claremont.edu. 


\title{
Continuum model of thin-film deposition and growth
}

\author{
Andrew J. Bernoff* \\ Department of Mathematics, University of Arizona, Tucson, Arizona 85721 \\ Seth Lichter \\ Department of Aerospace and Mechanical Engineering, University of Arizona, Tucson, Arizona 85721
}

(Received 5 December 1988)

\begin{abstract}
A continuum theory for the deposition and growth of solid films is presented. The theory is developed in a coordinate-independent manner and so incorporates the fully nonlinear physics. The evolution of the film is modeled in three steps. First, the adsorption of atoms in the incident beam is modeled as a ballistic process. Second, the random motion of the adatoms is treated as a diffusive process. Finally, sticking of adatoms to the film occurs as a Poisson process. The resulting system of differential equations is examined in several parameter limits. The diffusively dominated limit appears similar to zone 1 of the structure-zone model. Generically the surface slope develops discontinuities; these "kinks" play the role of grain boundaries. In the ballistically dominated case these kinks may be advected along the surface giving rise to columnarlike microstructures, as is observed in zone 2.
\end{abstract}

\section{INTRODUCTION}

While some features of film morphology are correlated to substrate temperature by the structure-zone model, ${ }^{1}$ prediction of film properties (such as film density, rate of growth, and column size) from deposition conditions is an actively pursued goal for both theoretical interest and technological importance. Past research has concentrated on explaining the microscopic dynamics of individual adatoms. Computer simulations serially deposit spheres or disks onto previously deposited particles to simulate the arriving beam flux. The adatoms then move over the surface according to some hopping algorithm. Continuum-like models also explicitly include microscopic variables such as adatom size. (See Bartholomeusz et $a{ }^{2}{ }^{2}$ for a review of previous research.)

In this paper the properties on the large scale are obtained by averaging over an ensemble of individual particles passing through a control volume on the surface. The local analysis is performed in a coordinateindependent fashion; consequently, the local balance then yields deposition equations in general coordinates that have the advantage of being fully nonlinear on the macroscopic scale. A simple ballistic argument yields a scattering cross section which determines the number of adatoms that are added to the surface layer and their velocity along the surface. The present paper attempts to track an atom from the beam until it is fixed to the film: it incorporates both a model of the initial collision with the interface and the adatom's mobility on the surface. It is hoped that this more comprehensive viewpoint will eventually explain a number of morphological features. In this paper some basic analysis of pertinent limiting cases is considered; a comprehensive categorization of behavior over the full parameter range is left for future efforts. In Sec. II the physical mechanisms are identified and incorporated into a mathematical formulation. An analysis of this model is presented in Sec. III. Results and comparison with experiment are in Sec. IV. In the Appendix details of the ballistic cross section are presented.

\section{FORMULATION}

In this section a local analysis of the adatom dynamics is used to deduce the deposition equations. The formulation incorporates the effects of incoming momentum, adatom motion, including convection and diffusion, and sticking. The incoming beam is assumed to be unidirectional and uniform. The incident atoms hit the substrate, and a portion of them join a loosely bound surface layer. A ballistic argument (presented in the Appendix) determines the portion of the beam flux which is not reevaporated. The flux rate onto a particular portion of the surface is then dependent upon the surface's orientation. ${ }^{3}$ The adatoms in this surface layer then move by convection and surface diffusion until they eventually stick, leading to accretion of the deposited film. Bulk diffusion is ignored. Finally, adhering to the surface is modeled as a Poisson process and consequently the deposition rate is proportional to the adatom concentration.

A length and time scale are chosen such that the variation on the substrate is initially order unity. Consider a location on the surface, $p$, and let the normal at this point be denoted by $\hat{\mathbf{n}}$ [Fig. 1(a)]. The incident adatom flux $\mathbf{J}$ will be assumed to be in direction $\widehat{\mathbf{J}}$ and of intensity $J$ :

$$
\mathbf{J}=J \hat{\mathbf{J}} \text {. }
$$

Moreover, the magnitude of the incident velocity, $V$, will also be assumed to be constant.

As the atoms strike the surface, some of them will be reflected off the surface, and some will be adsorbed into the surface layer. In the Appendix this process is examined in detail; here only two properties of the adatoms adsorbed at each point need to be known in detail-the 


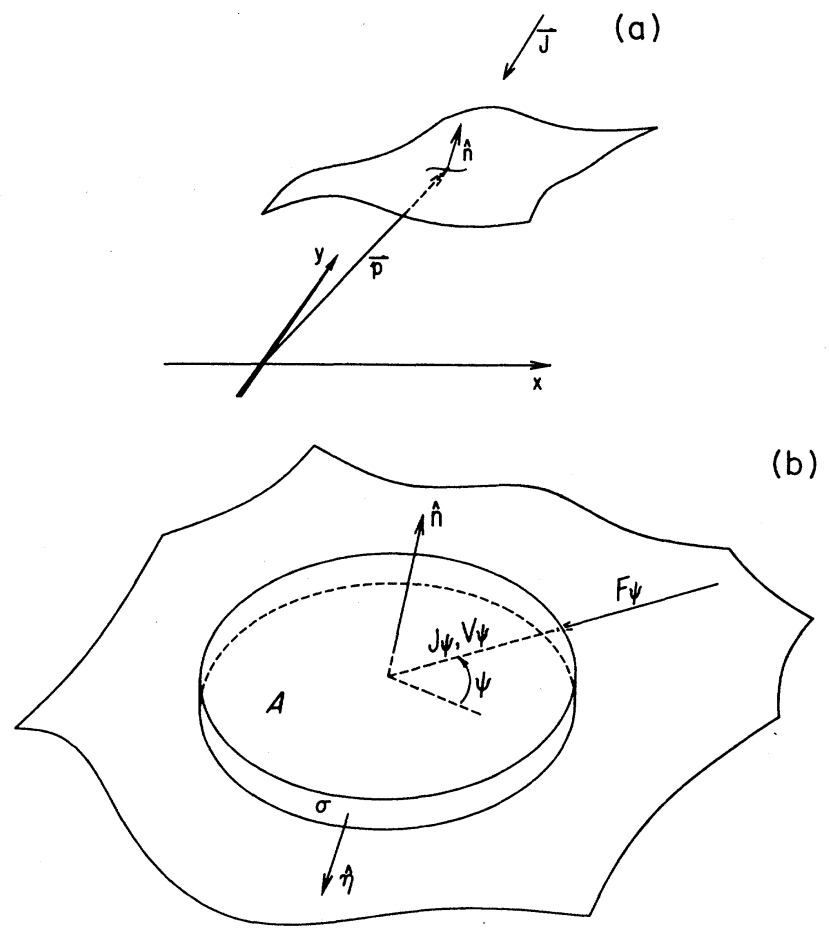

FIG. 1. (a) Schematic of the film surface. A point on the surface is denoted by $\mathbf{p}$. The normal to the surface is $\hat{\mathbf{n}}$. The incident flux $J$ is assumed uniform and unidirectional. (b) Control volume on the film surface. A flux balance through the top of the control surface $\mathcal{A}$ and edge $\sigma$ is shown. The normal to $\mathcal{A}$ is denoted $\hat{\mathbf{n}}$, whereas the normal along the edge $\sigma$ is denoted $\hat{\boldsymbol{\eta}}$. Flux and velocity along the surface $J_{\psi}$ and $V_{\psi}$ are defined as functions of location and polar angle on the tangent plane. At each point on $\sigma$ a directional flux $\mathscr{F}_{\psi}$ is defined. The flux in a direction $\hat{\psi}$ through $\sigma$ is given by $(\hat{\psi} \cdot \hat{\eta}) \mathcal{F}_{\psi}(\mathbf{p}(\sigma))$.

angular flux density of adatoms, $J_{\psi}$, and the mean velocity of the adsorbed adatoms, $V_{\psi}$. Define a polar angle $\psi$ in the tangent plane and a corresponding unit vector $\widehat{\psi}$. Then $J_{\psi}$ is the flux density per unit radian and $V_{\psi}$ is the mean velocity of the adatoms leaving in the $\hat{\psi}$ direction after collision.

The adatom travels along the surface in the $\hat{\psi}$ direction until it collides with another adatom or some local surface feature. The occurrence of this second collision is a Poisson-distributed process with time scale $1 / \gamma$. The length traveled before the second collision is then of order $V / \gamma$. The case when both these scales are small in comparison to the characteristic scales of the surface is considered, that is,

$$
\frac{1}{\gamma}, \frac{V}{\gamma} \ll<1
$$

Before the adatom undergoes a second collision, its velocity is determined by the scattering angle; consequent$1 y$, this population of adatoms will be called coherent. After the adatom has undergone a second collision, it will be assumed that it is scattered randomly. This population will be referred to as incoherent.
The equation of motion for the density of coherent adatoms, $c$, can now be computed by considering the flux balance in a small region, $\mathcal{A}$, of the surface. Denote the edge as $\sigma$ and the normal to the edge in the surface as $\hat{\boldsymbol{\eta}}$ [Fig. 1(b)]. Then the flux balance yields

$$
\begin{aligned}
\partial_{t}\left[\int_{\mathcal{A}} c d \mathcal{A}\right]= & \int_{\mathcal{H}} J_{n} d \mathcal{A}-\gamma \int_{\mathcal{A}} c d \mathcal{A} \\
& +\oint d \sigma \int_{0}^{2 \pi} d \psi(\hat{\boldsymbol{\psi}} \cdot \hat{\boldsymbol{\eta}}) \mathscr{F}_{\psi}(\mathbf{p}(\sigma)) .
\end{aligned}
$$

Here the left-hand side of (2.3) is the time rate of change of the volume of adatoms in $\mathcal{A}$. The terms on the righthand side are the flux through the top of $\mathcal{A}$, the loss due to deflection, and the flux through the edge of $\mathcal{A}$, respectively. The adsorbed flux density $J_{n}$ at a given point on the surface, $p$, can be computed by summing the fluxes scattered on the surface through all angles:

$$
J_{n}(\mathbf{p})=\int_{0}^{2 \pi} d \psi J_{\psi}(\mathbf{p}),
$$

and the incident flux adsorbed through the upper surface is then given by integrating $J_{n}$ over $\mathcal{A}$. The second term represents the loss of flux through deflection. This is modeled as a Poisson process; consequently, the time rate of loss of coherent adatoms is just a constant fraction $\gamma$ of the population. The last term in (2.3) represents the flux of adatoms through the edge, $\sigma$, computed by integrating the surface flux due to adatoms moving in a particular orientation through a boundary element. The flux of adatoms moving in the $\widehat{\psi}$ direction at a point on the boundary $\mathbf{p}(\sigma)$ can be computed as

$$
\mathcal{F}_{\psi}(\mathbf{p}(\sigma))=\int_{0}^{\infty} d r J_{\psi}\left(\mathbf{p}(\sigma)-r \widehat{\psi}, t-t^{\prime}\right) e^{-\gamma t^{\prime}},
$$

where the integrand is the contribution to the flux from a point a distance $r$ in the $-\hat{\psi}$ direction. The exponential term is the probability that a particle remains in the coherent population after a time $t^{\prime}$. The particles which strike the surface at a point, $p-r \hat{\psi}$, will arrive at the location $\mathbf{p}$ at time $t^{\prime}$ if

$$
r=V_{\psi} t^{\prime}
$$

Using the restrictions (2.2), the integral (2.5) can be evaluated to leading order as

$$
\begin{aligned}
\mathcal{F}_{\psi}(\mathrm{p}(\sigma)) & \approx \int_{0}^{\infty} d t^{\prime} V_{\psi}(\mathrm{p}(\sigma), t) J_{\psi}(\mathrm{p}(\sigma), t) e^{-\gamma t^{\prime}} \\
& =\frac{1}{\gamma} V_{\psi}(\mathrm{p}(\sigma), t) J_{\psi}(\mathrm{p}(\sigma), t) .
\end{aligned}
$$

Applying the divergence theorem to Eq. (2.3), and letting $\mathcal{A}$ shrink to a small area, yields the flux balance,

$$
c_{t}=J_{n}-\gamma c-\frac{1}{\gamma} \nabla_{s} \cdot\left(\mathbf{V}_{a} J_{n}\right)
$$

where the average velocity $\mathbf{V}_{a}$ has been defined as

$$
\mathbf{V}_{a}=\frac{\int_{0}^{2 \pi} d \psi J_{\psi} V_{\psi} \hat{\psi}}{J_{n}}
$$

The subscript $s$ on the vector operators is to indicate that they are acting along the surface defined by $\mathbf{p}$. The quantities $J_{n}$ and $\mathbf{V}_{a}$ are determined by the particular model used for ballistic scattering. These quantities are comput- 
ed for various cases in the Appendix.

Applying (2.2) shows that at leading order the solution to $(2.8)$ is

$$
c(\mathbf{p}, t)=\frac{1}{\gamma} J_{n}
$$

which can be interperted physically as the incident flux times the average time to deflection of an adatom.

Equation (2.8) describes the evolution of $c$ due to the coherent motion of ballistic scattering. The incoherent motion of adatoms caused by random scattering on the surface must also be included. It will be assumed that after an adatom in the surface layer is deflected the orientation of its velocity is determined randomly. Consequently, the motion of the particle can be modeled as a diffusive process.

The rate at which particles leave the coherent adatom concentration is $\gamma c$; they then become part of an incoherent adatom density $d$. Adatoms are removed from the incoherent state by sticking to the surface; this is again assumed to be a Poisson process with a mean lifetime $1 / \gamma^{\prime}$. The equation of motion for $d$ is now given by

$$
d_{t}=\gamma c-\gamma^{\prime} d+v \nabla_{s}^{2} d
$$

where $v$ is a diffusion constant.

As adatoms are deposited, the surface grows outward along its normal in proportion to the rate of deposition,

$$
\mathbf{p}_{t}=\gamma^{\prime} d \hat{\mathbf{n}} \text {. }
$$

This equation, together with (2.8) and (2.11), specifies the evolution of the surface.

To summarize, the adatom motion on the surface is due to a component which depends on the direction of the incident flux plus a randomly oriented diffusive component. The flux rate of particles which ultimately stick on the surface is given by $J_{n}$ and will be determined in the Appendix by considering the collision of an incident particle with the surface. The model equations governing the process of film growth are

$$
\begin{aligned}
& c_{t}=J_{n}-\gamma c-\frac{1}{\gamma} \nabla_{s} \cdot\left(\mathbf{V}_{a} J_{n}\right), \\
& d_{t}=\gamma c-\gamma^{\prime} d+v \nabla_{s}^{2} d, \\
& \mathbf{p}_{t}=\gamma^{\prime} d \hat{\mathbf{n}},
\end{aligned}
$$

where $\mathbf{p}$ is the location of the surface, $\hat{\mathbf{n}}$ is the surface normal, $c$ is the coherent adatom volume density, $d$ is the incoherent adatom volume density, $J_{n}$ is the adsorbed adatom flux, $\mathbf{V}_{a}$ is the average adsorbed adatom velocity, $1 / \gamma$ is the coherent adatom deflection rate, $1 / \gamma^{\prime}$ is the incoherent adatom sticking rate, and $v$ is the incoherent adatom diffusion constant.

\section{ANALYSIS}

\section{A. Conservation of integrated flux}

In this section it is shown how the integrated adsorbed incident flux relates to the integrated flux in the coherent and incoherent adatom populations and the increase in volume of the film. By considering the total incident flux onto the surface it is possible to determine an expression for the rate of growth of the film. Consider a domain, $\mathcal{A}$, with periodic or no-flux boundary conditions. Define the volume of previously deposited film below $\mathcal{A}$ as $\mathcal{V}$. Similarly, define the volume of incoherent and coherent adatoms on the surface as

$$
\begin{aligned}
& \int_{\mathcal{A}} c d S=\mathcal{C}, \\
& \int_{\mathcal{A}} d d S=\mathcal{D},
\end{aligned}
$$

respectively, where $d S$ is an element of surface area.

Integrating Eq. (2.13a) over the surface relates the total adsorbed incident flux,

$$
\not=\int_{\mathcal{A}} J_{n} d S
$$

to the evolution of $\mathcal{C}$,

$$
e_{t}+\gamma \mathcal{C}=\varnothing \text {. }
$$

Similarly, integrating $(2.13 b)$ relates the evolution of $C$ and $\mathcal{D}$,

$$
\mathscr{D}_{t}+\gamma^{\prime} \mathcal{D}=\gamma \mathcal{C}
$$

Finally, the time rate of change of the volume of the film can be related to the sticking of incoherent adatoms by taking the component of $(2.13 \mathrm{c})$ in the direction of the surface normal and integrating over the surface:

$$
\mathcal{V}_{t}=\gamma^{\prime} \circlearrowright
$$

The conservation of total mass can now be obtained by adding (3.3)-(3.5) together:

$$
\partial_{t}(\mathcal{C}+\mathcal{D}+\mathcal{V})=\not{\partial}
$$

Equations (3.3)-(3.6) demonstrate how the incident flux cascades through the various stages of the model. They allow the mean growth rate of the thin film to be determined as a function of the integrated adsorbed flux.

\section{B. Diffusion-dominated dynamics}

In this section the case when diffusion dominates the evolution of the interface will be considered. The mean diffusion length $L_{d}$ of an incoherent adatom can be approximated by considering $(2.13 \mathrm{~b})$,

$$
L_{d}=\left[\frac{v}{\gamma^{\prime}}\right]^{1 / 2} .
$$

Suppose the surface microstructure varies on a scale that is small compared to the diffusion length, that is, $L_{d} \gg 1$. In this case diffusion will be the dominant surface process. Equation (2.13b) will relax towards an equilibrium where $\nabla_{s} d$ is zero, that is, a state where the concentration of $d$ is uniform on the surface. This implies

$$
d \approx \frac{D}{\mathcal{A}}
$$

Solving (3.4) now yields

$$
d(t) \approx \frac{1}{\mathcal{A}} \int_{-\infty}^{t} \gamma \mathcal{C} e^{-\gamma^{\prime} t^{\prime}} d t^{\prime},
$$


or, applying (2.10),

$$
d(t) \approx \frac{1}{\mathcal{A}} \int_{-\infty}^{t} \not e^{-\gamma^{\prime} t^{\prime}} d t^{\prime} .
$$

In the limit when $\partial_{t} / \not \partial>\gamma^{\prime}$ this reduces to

$$
\gamma^{\prime} d \approx \frac{\partial}{\mathcal{A}},
$$

which is simply the average adsorbed flux.

If we define a new time scale

$$
\tau=\int_{0}^{t} \gamma^{\prime} d d t
$$

Eq. (2.13c) takes a particularly simple form,

$$
\mathbf{p}_{\tau}=\hat{\mathbf{n}} \text {. }
$$

Physically, the surface grows at a constant rate in the direction of its normal. In the limit defined by (3.11) the rate of growth is simply the average adsorbed flux rate. Equation (3.13) is an eikonal equation that commonly arises when considering the rays of a wave equation and can be solved implicitly with the method of characteristics. $^{4}$

It will be convenient to introduce a coordinate system for Eqs. (2.13); define the surface elevation $p$ measured above some plane with Cartesian coordinates $x_{i}=(x, y)$; then

$$
\mathrm{p}=(x, y, p(x, y, t)) .
$$

Equation (2.13c), which gives a Lagrangian description of the interface, can be converted to an Eulerian description. The normal at a point on the surface is given by

$$
\widehat{\mathbf{n}}=\frac{1}{\left(1+p_{x}^{2}+p_{y}^{2}\right)^{1 / 2}}\left(-p_{x},-p_{y}, 1\right) .
$$

If we denote by a dot the time derivative in the Lagrangian coordinate, the chain rule yields

$$
p_{\tau}=\dot{p}+p_{x} \dot{x}+p_{y} \dot{y},
$$

which, after using (3.13), yields

$$
p_{\tau}=\left(1+p_{x}^{2}+p_{y}^{2}\right)^{1 / 2} \text {. }
$$

Following Whitham ${ }^{4}$ an implicit solution to the initial value problem (3.17) can be found. It will be convenient in what follows to use index notation with the summation convention; define the gradient $\partial_{i}=\left(\partial_{x}, \partial_{y}\right)$. Suppose initially

$$
p\left(x_{i}\right)=P(x, y) \text { at } \tau=0 .
$$

Then at a later time $t$ the solution at a point $\check{x}_{i}=(\check{x}, \check{y})$ can be found in terms of $P(x, y)$,

$$
p(\check{x}, \check{y}, \tau)=P(x, y)+\frac{\tau}{\left(1+\partial_{k} P \partial_{k} P\right)^{1 / 2}},
$$

where $\check{x}_{i}$ is defined implicitly as a function of $x_{i}$ and $\tau$,

$$
\check{x}_{i}=x_{i}-\frac{\tau \partial_{i} P}{\left(1+\partial_{k} P \partial_{k} P\right)^{1 / 2}} \text {. }
$$

The implicit solution (3.20) allows some explicit special solutions; in particular, a plane will grow with a unit velocity normal to its surface and a spherical cap will remain spherical with a constantly increasing radius.

Because (3.20) is an implicit solution corresponding to characteristics, it admits the possibility of multivalued solutions. To determine if and when this happens it is necessary to decide if (3.20) provides a continuous bijection from $x_{i}$ to $\check{x}_{i}$. This will be the case if the Jacobian $Q$ between the two coordinates is always nonzero. Using (3.20) to compute the Jacobian yields the determinant

$$
Q=\left|\frac{\partial \check{x}_{i}}{\partial x_{j}}\right|=\left|\delta_{i j}-\tau M_{i j}\right|,
$$

where the $\delta_{i j}$ is the Kronecker $\delta$ function and

$$
M_{i j}=\frac{\partial_{i j} P\left(1+\partial_{k} P \partial_{k} P\right)^{1 / 2}-\partial_{i} P \partial_{k} P \partial_{j k} P}{\left(1+\partial_{k} P \partial_{k} P\right)^{3 / 2}} .
$$

To understand (3.22) requires a basic knowledge of differential geometry. ${ }^{5}$ The determinant and trace of $M_{i j}$ are the Gaussian curvature and twice the mean curvature, respectively. This implies that the eigenvalues of $M_{i j}$ are exactly the principal curvatures, $\kappa_{1}, \kappa_{2}$ of the surface. Geometrically, $1 / \kappa_{1}, 1 / \kappa_{2}$ correspond to the maximum and minimum radius of the osculating circle at a point on $P$. From (3.21) it follows that $Q$ will vanish when

$$
\tau=\min _{\left\{x_{i}\right\}}\left(\frac{1}{\kappa_{1}}, \frac{1}{\kappa_{2}}\right) .
$$

Note that if $\kappa_{1}, \kappa_{2}$ are never greater than zero, the initial condition is convex, and the solution never breaks down. However, if the initial condition is nonconvex anywhere, at least one of the curvatures will be positive and the solution will eventually break down.

The form of this breakdown can be deduced by noting that the gradient of (3.17) is a first-order semilinear hyperbolic system for $\partial_{i} p$. Consequently, the gradient will develop shocks (discontinuities) and $p$ will develop "kinks" (discontinuities in the derivative of $p$ ). In two dimensions these kinks will occur at isolated points; in three dimensions they will occur on curves on the surface. A description of the motion of these kinks is needed for (3.17) to provide a complete description of the dynamics. Fortunately, physical insight provides a unique solution for the motion of the kinks. The equations are invariant under multiplying $x_{i}, p$, and $\tau$ by a constant; from this it follows that the velocity is dependent only on the slope of the tangent plane on each side of the kink. The isotropy of space now implies that the kink must propagate along the angle bisector of the dihedral angle formed by the tangent planes at the kink (Fig. 2). This rule together with the characteristic description given by (3.19) and (3.20) is sufficient to describe the dynamics. A sample numerical evolution of a two-dimensional initial condition is shown in Fig. 3.

In three dimensions other phenomena can occur; the kinks correspond to boundaries between grains. If the evolution from an initial condition corresponding to a rough substrate is considered, various bifurcations can occur. The initial appearance of the kink will still occur 


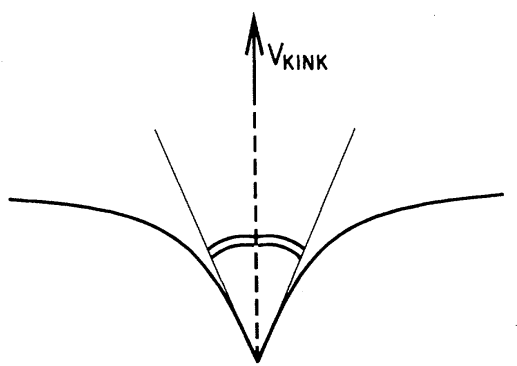

FIG. 2. Propagation of a kink along the angle bisector.

at a point corresponding to the highest curvature of a concavity. After formation the kink will become a line of discontinuity. Pairs of lines of kinks can collide, creating triple points where three grains meet (Fig. 4). These triple points can also occur by the appearance of a new discontinuity in curvature transverse to an existing line of kinks. The evolution of this triple conjunction is again easily prescribed; it must propagate along the line given by the intersection of the angle bisectors of the three dihedral angles created by the tangent planes at the point. In this way an initially variable topography will lead to a system of kinks connected at triple points, resembling the networks of grains seen in thin-film growth.

\section{Ballistic-dominated dynamics}

A second special case of interest is when ballistic effects dominate the effect of diffusion. The limit considered here is when the diffusion length is much smaller than the characteristic length in the problem, that is, $L_{d} \ll 1$. In addition, it will be assumed that the deposi-

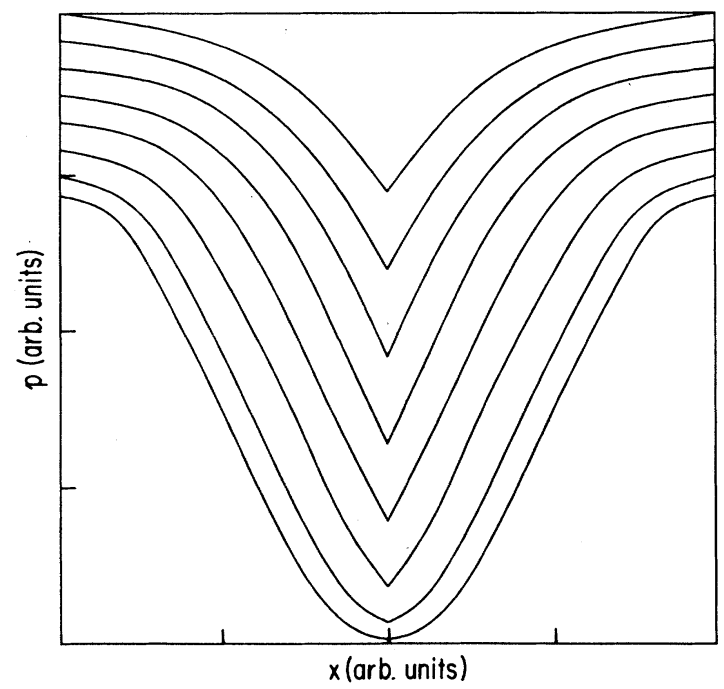

FIG. 3. Sample evolution for the eikonal equation. The evolution of an initial condition corresponding to a sinusoidal substrate is shown at evenly spaced time intervals. The concavity leads to the formation of a kink-type discontinuity. The kink propagates along the angle bisector of the tangent plane (cf. Fig. 2). Note that surface roughness decreases with increasing time.

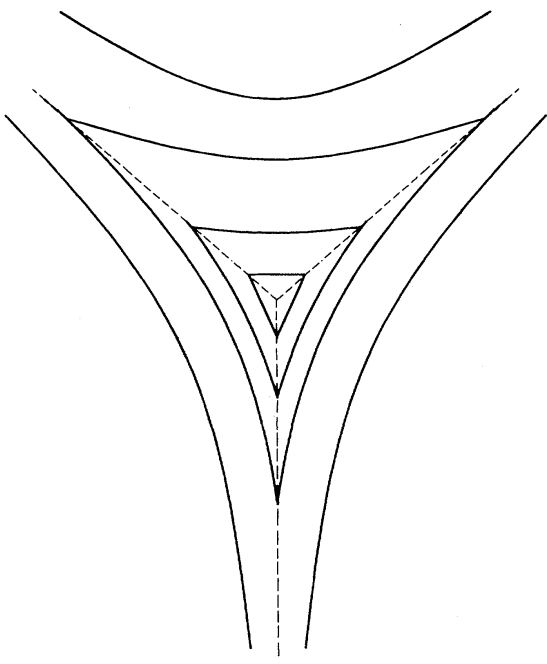

FIG. 4. Triple points. In three dimensions three kinks can originate from a single point. Near the triple point the surface can be approximated by the tangent planes to the three grains between the three kinks. The motion of the point is along the angle bisector of the dihedral angles formed by the tangent planes. The networks of kinks connecting triple points form a grainlike structure which is similar to that observed experimentally.

tion time is short compared to the surface growth rate, that is, $1 / \gamma^{\prime} \ll<$. In this case, at leading order

$$
\gamma^{\prime} d \approx \gamma c \approx J_{n} \text {. }
$$

Equation $(2.13 \mathrm{c})$ now reduces to

$$
\mathbf{p}_{t}=J_{n} \hat{\mathbf{n}} \text {. }
$$

It will be convenient to consider a two-dimensional evolution for Eq. (3.25). Following (3.14) the height of the surface is defined as

$$
\mathbf{p}=(x, y, p(x, t)),
$$

where the dependence of $p$ on $y$ has been suppressed. In addition, it is more illuminating to introduce $\phi$, defined as the angle the surface normal makes with the direction of incoming flux. It will be assumed that $\widehat{\mathbf{J}}=-\widehat{\mathbf{z}}$, which implies

$$
\cos \phi=-\widehat{\mathbf{J}} \cdot \hat{\mathbf{n}}=\frac{1}{\left(1+p_{x}^{2}\right)^{1 / 2}} .
$$

Equation (3.27) and the restriction that $\phi$ is positive when the slope of $p$ is positive specify $\phi$ in terms of $p$.

The assumption $\hat{\mathbf{J}}=-\widehat{\mathbf{z}}$ is not at all restrictive; the isotropy of our formulation guarantees that looking at vertical flux on a slanted surface is identical to looking at flux incident from the same angle on a flat surface. Using (3.27), $p$ can be eliminated in favor of $\phi$ in Eq. (3.25),

$$
\phi_{t}=\cos ^{2}(\phi) \partial_{x}\left[J_{n} \sec (\phi)\right] \text {. }
$$

From the ballistic results in the Appendix, it is clear that $J_{n}$ depends only on $\phi$, so Eq. (3.28) can be rewritten as 


$$
\phi_{t}+U(\phi) \phi_{x}=0,
$$

where

$$
U(\phi)=-\left[\left(J_{n}\right)_{\phi} \cos (\phi)+J_{n} \sin (\phi)\right] .
$$

Equation (3.29) is a wave equation. Any initial condition with a variation in $\phi$ and $U$ should cause the formation of shocks. Note that a shock corresponds to a discontinuity in $\phi$; from (3.27) it follows that this corresponds to a kink in the surface of the film as described by p.

Figure 5 shows $U$ graphed for various ballistic models. Physical symmetry implies that the velocity is an antisymmetric function of $\phi$. It is curious to note in the three-dimensional hard-sphere case that $U$ is zero, and Eq. (3.29) corresponds to growth at a constant rate towards the source. For the other three-dimensional cases, note that $U$ is negative for $\phi>0$, and decreases to a minimum and tends to zero as $\phi$ tends to $\pi / 2$. For the two-dimensional case, hard-sphere $U$ changes sign; this will lead to qualitatively different dynamics. This should be taken as a caution against using two-dimensional ballistic models.

When Eq. (3.29) becomes invalid due to shock formation, it is essentially because of the formation of high gradients in $\phi$. Because derivatives become large, higherorder terms in the equation become important. This will lead to a regularization of the wave equation.

The next-higher-order contributions due to corrections to (3.24) can easily be computed. Substituting (3.24) into (2.20) yields

$$
\gamma^{\prime} d \approx J_{n}+v \nabla_{s}^{2} d-d_{t}-c_{t}-\frac{1}{\gamma} \nabla_{s} \cdot\left(\mathbf{V}_{a} J_{n}\right),
$$

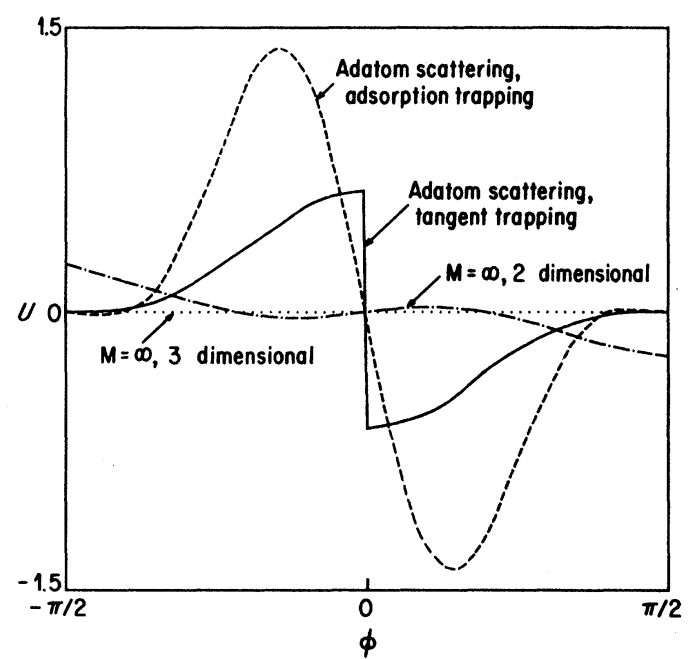

FIG. 5. Velocity $U$ as a function of $\phi$ for various models. Note that the three-dimensional hard-sphere model is identically zero. The two-dimensional hard-sphere model exhibits a change of sign not seen in any of the three-dimensional models. Presumably the solutions for finite-mass ratios $(\boldsymbol{M})$ greater than unity lie between the $M=1$ and $\infty$ cases and also exhibit a single maximum. which, resubstituting (3.24), and using (3.27) and (3.29), yields

$$
\begin{aligned}
\gamma^{\prime} d \approx & J_{n}+\left[\left[\frac{1}{\gamma^{\prime}}+\frac{1}{\gamma}\right] U\left(J_{n}\right)_{\phi}-\frac{\cos (\phi)}{\gamma} \partial_{\phi}\left(\mathbf{V}_{a} J_{n}\right)\right] \phi_{x} \\
& -\frac{v}{\gamma^{\prime}} \cos (\phi) \partial_{x}\left[\cos (\phi) \partial_{x}\left(J_{n}\right)\right]
\end{aligned}
$$

The second and third terms on the right-hand side represent corrections to the approximation made by (3.24). By balancing the leading-order term $J_{n}$ against each of these terms, the shock width produced by each of the regularizations can be deduced. Note that the second term has a single $x$ derivative; it corresponds to a dissipative regularization. This term becomes important on the shock length scale $L_{s}$, which is dimensionally approximated by

$$
L_{s} \sim J_{n}\left(\frac{1}{\gamma}+\frac{1}{\gamma^{\prime}}\right)+\frac{V}{\gamma} .
$$

Note that in general this scale is on the order of the ballistic scattering distance; consequently, the arguments used to derive (2.13a) are no longer valid. If this term leads to the regularization, a detailed examination of the ballistic effects in this region will be needed.

If the third term in (3.32) leads to the regularization of the shock, the shock width $L_{s}^{\prime}$ will be

$$
L_{s}^{\prime} \sim\left(\frac{v}{\gamma}\right)^{1 / 2}=L_{d},
$$

which is exactly the diffusion length. Note that the term has two $x$ derivatives, corresponding to a dispersive regularization.

To understand qualitatively the dynamics of these two regularizations, the special case where $\phi$ is nearly constant is considered. Let

$$
\phi(x, t)=\phi_{0}+\epsilon \Phi(x, t),
$$

where $\epsilon \ll 1$. Consider a change of variables into a frame moving at the phase velocity $U\left(\phi_{0}\right)$, and on a slow time scale,

$$
\begin{aligned}
& X=x-U\left(\phi_{0}\right) t, \\
& T=\epsilon t .
\end{aligned}
$$

Then Eq. (3.29) can be expanded as

$\Phi_{T}+U^{\prime}\left(\phi_{0}\right) \Phi \Phi_{x}+F\left(\phi_{0}\right) \Phi_{x x}+G\left(\phi_{0}\right) \Phi_{x x x}+\ldots=0$,

where

$$
\begin{gathered}
F\left(\phi_{0}\right)=\frac{\cos \left(\phi_{0}\right)}{\epsilon}\left[\left[\frac{1}{\gamma^{\prime}}+\frac{1}{\gamma}\right] U\left(J_{n}\right)_{\phi}\right. \\
\left.-\frac{\cos \left(\phi_{0}\right)}{\gamma} \partial_{\phi}\left(\mathbf{V}_{a} J_{n}\right)\right], \\
G\left(\phi_{0}\right)=\frac{v}{\epsilon \gamma^{\prime}} \cos ^{3}\left(\phi_{0}\right)\left(J_{n}\right)_{\phi} .
\end{gathered}
$$


In the case when $L_{s} \gg L_{d}$, the $\Phi_{x x}$ term will be the dominant regularization. In this case Eq. (3.36) is Burger's equation. When $F\left(\phi_{0}\right)$ is negative, the shock will have a width of $O\left(L_{s} / \epsilon\right)$, and characteristics will flow uniformly into the shock. This leads to the decay of the shock and for large time the solution tends to a uniform constant. When $F\left(\phi_{0}\right)$ is positive the diffusion is acting in a negative sense, and the equation is ill posed: any small disturbance will tend to focus and blow up in a finite amount of time. Presumably this blowup might saturate if higher-order terms are included. However, any model with this asymptotic limit presumably exhibits highly chaotic dynamics, similar to what is seen in the Kuramoto-Sivashinsky equation.

In the case when $L_{s}<<L_{d}$, the $\Phi_{x x x}$ term will be the dominant regularization. In this case Eq. (3.36) is the Korteweg-de Vries $(\mathrm{KdV})$ equation. $\mathrm{KdV}$ is well known for exhibiting soliton-type solutions: lumplike objects which are exponentially localized and translate without change. The width of the soliton would be $O\left(L_{d} / \epsilon\right)$. Note that in terms of the surface elevation $p$ a soliton would be a step separating two regions of constant slope (Fig. 6). As $\epsilon$ increases, presumably this would correspond to a narrow region of width $L_{d}$ separating two flat regions, and translating with speed of $U\left(\phi_{0}\right)$.

\section{RESULTS AND CONCLUSIONS}

In this paper a formalism is described for deriving a continuum model of thin-film growth. The deposition is broken into three processes: a ballistic scattering model is used to determine adsorption onto the surface; the random walk of the adatoms on the surface is described by a diffusion equation; and sticking of the adatoms to the surface is described as a Poisson process. Because the model is formulated in a coordinate-independent fashion, the local dynamics, which are basically linear, lead to a fully nonlinear model of the large-scale evolution. This supersedes the earlier work of Lichter and Chen, ${ }^{6}$ who only considered linear perturbations to a flat film.

The proportion of incident atoms adsorbed can be

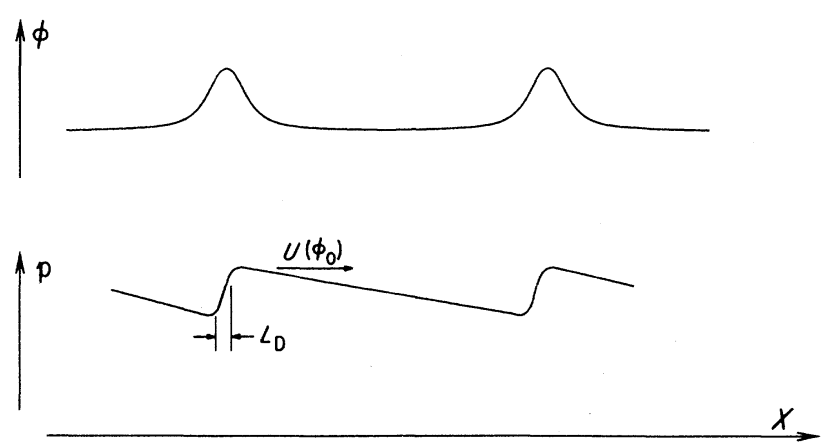

FIG. 6. Soliton propagation. Equation (3.36) has solitonlike solutions in the dispersively regularized limit. The soliton in $\phi$ corresponds to a step in surface elevation of width $L_{d}$ between regions of uniform slope. The step, like the soliton, will propagate with a speed $U\left(\phi_{0}\right)$. computed from the ballistic models described in the Appendix. This in turn allows the film growth rate to be computed and compared to experiment.

In the case when surface diffusion is the dominant process, an eikonal equation has been derived to describe the growth of the film. The film locally grows at a constant rate normal to its surface. The eikonal equation has a special solution of note; a sphere or spherical cap will grow in a self-similar fashion with a constantly growing radius. This means a local bulge in substrate leads to a conelike solution. In addition, an imperfection seeding the medium may also cause a conical growth pattern.

The regime in which this behavior is expected is given by the mean diffusion length $L_{d} \gg 1$. This parameter can be expected to depend exponentially on the inverse of the temperature; ${ }^{7}$ this implies that low substrate temperatures will correspond to a large diffusion length. The cone-shaped microstructure with spherical caps corresponds to characteristic morphology of zone 1 of the structure zone model. ${ }^{1}$

In addition, the eikonal equations predict the formation of kinklike discontinuity of slopes. A network of such kinks is expected for an initially rough substrate. This network of grain boundaries seems characteristic of experimental observations. ${ }^{2}$

The model is also examined in the case when ballistic advection is much greater than diffusion. Here the characteristic equation describing the film's growth is a first-order wave equation. For three-dimensional hardsphere scattering, the phase velocity of the wave equation is zero; consequently, the solution will always grow in a self-similar fashion towards the incident flux. However, energy loss to the atomic lattice is expected ${ }^{8}$ after the substrate has been coated with the incident species. This energy loss is modeled by using a ballistic cross section with a spherical particle with a finite effective mass. This leads to a nonuniform phase velocity for the governing wave equation. This nonuniformity will lead to shock formation which once again manifests itself as a discontinuity in the surface slope. These shocks will propagate across the film; their tracks will leave columnarlike deposition, as observed in zone 2 of the structure-zone model. ${ }^{1}$

The details of the shock structure depend upon the higher-order corrections to the wave equation. Two candidates for regularizing the wave equation have been identified. When surface diffusion is small compared to the coherent scattering distance of an adatom, the regularization is dissipative, and a Burger's-type equation is an appropriate model for the evolution. In this case shocks are localized and propagate. ${ }^{4}$ When the diffusion length is longer than the coherent scattering length, the appropriate regularization is dispersive. Here the canonical governing equation is Korteweg-deVries. This allows the possibility of solitonlike solutions corresponding to a steplike surface geometry. These equations undoubtably exhibit a rich and varied dynamics depending on the parameter regimes examined, and consequently they are left for future study.

Note that in the diffusion-dominated limit, a flat surface will grow in a direction normal to its surface, in- 
dependent of the direction of the incident flux. In the ballistically dominated limit, when three-dimensional hard-sphere scattering is considered, the surface grows at a constant rate in the direction of the incident flux, independent of the surface normal. It seems logical to assume that when these two effects are comparable the surface will grow at some angle between the normal and the direction of incident flux. Experiments observe that the angle of columnar growth in zone 2 of the structure zone model $^{1}$ is intermediate between these two extremes, and follows the empirical relationship known as the tangent rule. ${ }^{3}$ Our model suggests that this effect may be due to a balance of surface diffusion and ballistic effects.

\section{ACKNOWLEDGMENTS}

Many discussions with Ursula Gibson and the support of the U.S. Air Force Office of Scientific Research under Contract No. F 49620-86-C-0123 are gratefully acknowledged.

\section{APPENDIX: BALLISTIC COMPUTATION OF FLUX CROSS SECTIONS}

In this Appendix a formalism for using the ballistics of a two-sphere interaction to derive a flux and velocity cross section on the surface of the film is presented. Consider an incident spherical particle striking a surface with a rough atomic scale. Assume that the orientation of the surface on the atomic scale is basically random. This case corresponds to the incident particle being scattered from a spherical body because the orientation of the normal to a sphere is isotropic in space. In addition, the film will have a certain degree of elasticity. This will be modeled by defining a mass ratio $M$ between the mass of the incoming adatom and the effective mass of the particle struck in the film. The limit $M \rightarrow \infty$ corresponds to scattering off a fixed hard sphere, whereas $M=1$ corresponds to striking an unbound adatom. The study is restricted to $M$ between these limits.

Using conservation of momentum and energy allows us

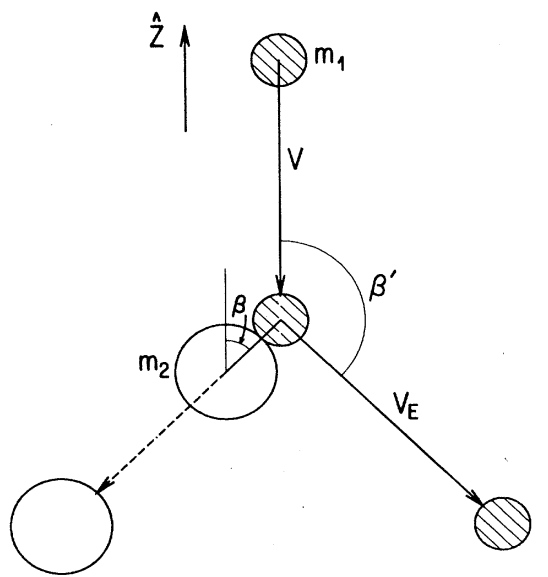

FIG. 7. Schematic of a scattering collision. The incident particle $m_{1}$ with initial velocity $V$ strikes the stationary particle $m_{2}$ at an impact angle $\beta$. It is scattered through an angle $\beta^{\prime}$ with an exit velocity $V_{E}$. The ballistic cross section can be examined, and $\beta^{\prime}$ and $V_{E}$ can be computed as a function of $V, \beta$, and the mass ratio $M=m_{2} / m_{1}$. to relate the exit velocity $V_{e}$ and scattering angle $\beta^{\prime}$ as a function of the incident velocity $V$, and impact angle $\beta$ (Fig. 7). The velocity ratio can be computed as

$$
\frac{V_{e}}{V}=\frac{\left[M^{2}-\sin ^{2}\left(\beta^{\prime}\right)\right]^{1 / 2}-\cos \left(\beta^{\prime}\right)}{1+M} .
$$

The relation between the impact angle and scattering angle can only be written implicitly:

$$
\operatorname{cotan}\left(\beta^{\prime}\right)=\frac{\cos (2 \beta)-1 / M}{\sin (2 \beta)} .
$$

The incident flux is assumed to be arriving vertically, that is,

$$
\widehat{\mathbf{J}}=-\hat{\mathbf{z}} \text {. }
$$

The scattering from a unit sphere at the origin will be computed. Define spherical polar coordinates, where $\alpha, \beta$ are the azimuthal angle measured from $\hat{\mathbf{x}}$ and the declination from $\hat{\mathbf{z}}$, respectively. The projection along the $z$ axis is the unit circle. Define the radial component, $r$, in the $x-y$ plane,

$$
r=\left(x^{2}+y^{2}\right)^{1 / 2}=\sin \beta
$$

then the differential flux density, $d J$, is given by the flux times the area element in the plane divided by the total area of the projection,

$$
d J=\frac{J}{\pi} r d r d \alpha \text {. }
$$

Notice that the integral over the entire cross section simply yields $J$. Substituting (A4) into (A5) yields the cross section as a function of $\alpha, \beta$ :

$$
d J=\frac{J}{\pi} \sin (\beta) \cos (\beta) d \alpha d \beta
$$

To compute the flux scattered out at various angles, the scattering angle must be known; assume that the particle leaves in a direction $\alpha^{\prime}, \beta^{\prime}$. For the geometry considered here the azimuthal angle remains the same $\left(\alpha^{\prime}=\alpha\right)$ and Eq. (A2) relates the scattering angle $\beta^{\prime}$ to the impact angle $\beta$. The differential cross section for the scattered particles can now be written as

$$
d J=\frac{J}{\pi} \sin (\beta) \cos (\beta)\left[\frac{d \beta}{d \beta^{\prime}}\right] d \alpha^{\prime} d \beta^{\prime},
$$

where it is understood that $\beta$ is a function of $\beta^{\prime}$

Finally, it is desirable to compute the differential cross section in a spherical coordinate system oriented along the normal to the surface, $\hat{\mathbf{n}}$. Let the angle between the normal and $\hat{\mathbf{z}}$ be denoted by $\phi$; without loss of generality it can be assumed that the normal lies in the $x-z$ plane and that its projection on $\hat{\mathbf{x}}$ is negative. Define an angle of declination from the normal, $\chi$, and an azimuthal angle, $\psi$, which is measured from the $\hat{\mathbf{x}}$ direction. The twocoordinate systems are then related by

$$
\begin{aligned}
& \cos (\chi)=\cos (\beta) \cos (\phi)-\sin (\phi) \sin (\beta) \cos (\alpha), \\
& \sin (\chi) \cos (\psi)=\cos (\beta) \sin (\phi)+\cos (\phi) \sin (\beta) \cos (\alpha),
\end{aligned}
$$




$$
\sin (\chi) \sin (\psi)=\sin (\beta) \sin (\alpha) .
$$

Equations (A8) can now be used to convert (A7) into an expression in terms of $\chi, \psi$ :

$d J=\frac{J}{\pi}\left(\frac{d \beta}{d \beta^{\prime}}\right) \cos (\beta) \sin (\beta)\left(\frac{\sin (\chi)}{\sin \left(\beta^{\prime}\right)}\right) d \psi d \chi$,

where $\beta$ and $\beta^{\prime}$ must be eliminated in terms $\psi, \chi$.

Finally, the angular cross section can be obtained by integrating over the range of $\chi$ which are deflected onto the surface. In addition, $d J$ is the differential cross section per unit area in the $x-y$ plane. In the paper the incident flux per unit area on the surface of the film is desired; the ratio of these areas, which is given by the dot product of the normal to the surface and $\hat{\mathbf{z}}$, is simply $\cos (\phi)$. Consequently

$$
J_{\psi}=\cos (\phi) \int_{\chi} d J \text {. }
$$

In the remainder of this appendix the cross sections for various particular cases are computed. Two types of surface trapping are considered: tangent trapping, where only the adatoms deflected nearly tangent to the surface will not be revaporated and will ultimately stick, and adsorption trapping, where any adatom with a velocity directed into the surface after scattering is assumed to be entrapped by the surface adatom layer.

\section{Three-dimensional hard-sphere scattering: Tangent and adsorption trapping}

This corresponds to the case of an infinite mass ratio $(M=\infty)$. From (A1) and (A2) it follows that

$$
V_{e}=V, \quad \beta^{\prime}=2 \beta \text {. }
$$

Substituting into (A7) yields

$$
d J=\frac{J}{4 \pi} \sin \left(\beta^{\prime}\right) d \alpha^{\prime} d \beta^{\prime}=\frac{J}{4 \pi} d S^{\prime},
$$

where $d S^{\prime}$ is the element of surface area. This implies that the adatoms are scattered with a constant density per solid angle. Moreover, the cross section is independent of surface orientation.

To evaluate the integral (A10), first consider the tangent trapping case. Note that scattering with $\chi=\pi / 2$ corresponds to leaving tangent to the surface. Define a small angle $\Delta$, corresponding to the angular cross section around $\chi=\pi / 2$ that is trapped by the surface. In this case $J_{\psi}$ can be approximated by

$$
\left.J_{\psi} \approx \Delta d J\right|_{\chi=\pi / 2}
$$

This allows the computation of

$$
J_{\psi}=\frac{J \Delta}{4 \pi} \cos (\phi),
$$

and (2.4) yields

$$
J_{n}=\frac{J \Delta}{2} \cos (\phi) \text {. }
$$

In the absorption case all adatoms with $\pi / 2<\chi<\pi$ will be added to the surface layer. In this case,

$$
J_{\psi}=\frac{J}{4 \pi} \cos (\phi)
$$

and

$$
J_{n}=\frac{J}{2} \cos (\phi)
$$

\section{Two-dimensional hard-sphere scattering: Tangent trapping}

If the two-dimensional hard-sphere case is considered, isotropic scattering is no longer seen; this should be taken as an omen that two-dimensional ballistic models may have drastically different results from the threedimensional cases they simulate.

In two dimensions the differential flux density is given by

$$
d J=\frac{J}{2} d r=\cos (\beta) d \beta
$$

Substituting (A11) yields

$$
d J=\frac{J}{4} \cos \left(\frac{\beta^{\prime}}{2}\right) d \beta^{\prime} .
$$

Note that $\chi=\beta^{\prime}+\theta$. In this case $J_{\psi}$ is replaced by $J_{+}$ and $J_{-}$, defined as the density of adatoms scattered forward and backward, respectively. Only the tangent trapping case will be computed here; for it,

$$
J_{ \pm}= \pm \frac{J \Delta}{4} \cos \left(\frac{\pi}{4} \pm \frac{\phi}{2}\right) \cos (\phi) .
$$

The integrated flux is defined as

$$
J_{n}=J_{+}+J_{-}=\frac{J \Delta}{4 \sqrt{2}} \cos \left(\frac{\phi}{2}\right) \cos (\phi) .
$$

\section{Adatom scattering: Tangent trapping}

In this case an adatom scattering off a particle of equal mass is considered; that is, $M=1$. Considering relationship (A2) shows that

$$
\beta^{\prime}=\beta+\frac{\pi}{2} \text {. }
$$

Consequently, $\pi / 2<\beta^{\prime}<\pi$, and for this range the velocity is given by

$$
V_{\psi}=-\cos \left(\beta^{\prime}\right) \text {. }
$$

Substituting into (A7) yields

$$
d J=-\frac{J}{\pi} \cos \left(\beta^{\prime}\right) \sin (\chi) d \chi d \psi \text {. }
$$

Judicious application of Eqs. (A8) yields

$$
\cos \left(\beta^{\prime}\right)=\cos (\chi) \cos (\theta)+\sin (\chi) \cos (\psi) \sin (\theta)
$$

and applying (A8) yields

$$
J_{\psi}=-\frac{2 J \Delta}{\pi} \cos (\psi) \cos (\phi)|\sin (\phi)| .
$$

To evaluate the integrated flux, (A26) is integrated over $\psi$ 
for $\beta>\pi / 2$, to yield

$$
J_{n}=\frac{2 J \Delta}{\pi}|\sin (\phi)| \cos \phi
$$

\section{Adatom scattering: Adsorption trapping}

In the case of an adsorption trapping, the density given by (A24) must be integrated over the sector where $\beta^{\prime}, \chi>\pi / 2$. This yields, after modest calculation,

$$
J_{n}=J \cos (\phi)\left[\frac{\cos ^{3}(\phi)+\cos (\phi)}{2}\right.
$$

$$
\left.+\frac{\sin (\phi)}{2 \pi}[2 \phi+\sin (2 \phi)]\right] \text {. }
$$

*Present address: Department of Mathematics, University of California, Berkeley, CA 94720.

${ }^{1}$ B. A. Movchan and A. V. Demchishin, Fiz. Met. Metalloved. 28, 83 (1969).

${ }^{2}$ B. J. Bartholomeusz, K.-H. Muller, and M. R. Jacobson, Proc. SPIE 821 (1987).

${ }^{3}$ A. G. Dirks and H. J. Leamy, Thin Solid Films 47, 219 (1977).

${ }^{4} \mathrm{G}$. B. Whitham, Linear and Nonlinear Waves (Wiley-
Interscience, New York, 1974).

${ }^{5}$ M. P. Do Carmo, Differential Geometry of Curves and Surfaces (Prentice-Hall, Englewood Cliffs, 1976).

${ }^{6}$ S. Lichter and J. Chen, Phys. Rev. Lett. 56, 1396 (1986).

${ }^{7}$ W. K. Burton, N. Cabrera, N. Cabrera, and F. C. Frank, Philos. Trans. R. Soc. London, Ser. A 23, 299 (1951).

${ }^{8}$ J. E. Lennard-Jones, Proc. R. Soc. London, Ser. A 163, 127 (1937). 